Results: Arterial umbilical cord copeptin concentrations were consistently higher that matched venous ones $(p<0.001)$ but copeptin concentrations were closely related $(R s=0.825, p$ $<0.001)$. Exceedingly high copeptin concentrations were observed after vaginal birth in umbilical cord venous (median [5-95\% range]: 793 [6-4836] $\mathrm{pmol} / \mathrm{L}$ ) and arterial plasma [1610 [85-5000] pmol/L). In addition, copeptin concentrations in umbilical venous and arterial blood were negatively related with birth acidosis $(\mathrm{pH}, \mathrm{Rs}=-0.578$ and -0.639 , both $p<0.001$ ). Postnatal body weight was associated with increased copeptin concentrations at day 3 (Rs $=0.438, p<0.001$ ) and was inversely related with copeptin concentrations at birth in umbilical venous and artery plasma (Rs $=-0.289$ and -0.309 , both $p$ $=0.001$ ).

Conclusion: Vaginal birth is associated with a huge release of AVP/copeptin. Umbilical cord copeptin concentrations exceed values published so far, including those in critically ill adult patients with myocardial infarction, shock, or brain injury.

96

\section{THE LEUKOENCEPHALOPATHIES OF CHILDHOOD: FINDINGS FROM A NATIONAL PROSPECTIVE STUDY}

C.M. Verity ${ }^{1}$, A.M. Winstone ${ }^{1}$, L. Stellitano ${ }^{1}$, A. Maw ${ }^{1}$, M.S. van der Knaap ${ }^{2}$

${ }^{1}$ PIND Surveillance Group, Addenbrookes Hospital, Cambridge, UK, ${ }^{2}$ VU University Medical Center, Amsterdam, The Netherlands

Background and aims: To report the distribution of leukoencephalopathies in children identified by this prospective population-based study.

Methods: 2802 children with suspected progressive intellectual and neurological deterioration (PIND) were notified via the British Paediatric Surveillance Unit (BPSU) between 1997 and 2009. An expert group reviewed clinical data and confirmed diagnoses where possible. An independent expert reviewed the available scans of children with unclassified leukoencephalopathies.

Results: 1132 of the PIND children had an underlying diagnosis and of these 295 had one of the leukoencephalopathies.The distribution of diagnosed leukoencephalopathies was as follows: metachromatic leukodystrophy 62, X-linked adrenoleukodystrophy 60, Krabbe disease 39, Canavan disease 16, Alexander disease 13, leukoencephalopathy with vanishing white matter 12, Aicardi-Goutieres syndrome 12, PelizaeusMerzbacher disease 11, megalencephalic leukoencephalopathy with subcortical cysts 8, multiple sulphatase deficiency 6 , multiple sclerosis 3 , peroxisomal bifunctional D-protein deficiency 2 , peroxisomal biogenesis defect 1 .

There were 50 children in the unclassified leukoencephalopathy group. In 32 the scans were reviewed and 19 were placed in a hypomyelination sub-group, the other 13 remaining in the unclassified leukoencephalopathy group with the 18 whose scans could not be reviewed.

Conclusions: Leukoencephalopathies comprised $26 \%$ of PIND children with a known diagnosis. This population based study gives the relative frequency of the different leukoencephalopathies, providing an aid to diagnosis in children with abnormal white matter on brain imaging. After rigorous investigation $16 \%(50 / 295)$ remained in an unclassified group, but this situation is evolving.

Acknowledgements: Thanks to the English Department of Health for funding [121/6443], the BPSU and the PIND Expert Group.

\section{7}

\section{DISTRIBUTION OF MICROGLIA (MG) IN THE IMMATURE BRAIN WITH ISOLATED GERMINAL MATRIX/INTRAVENTRICULAR HAEMORRHAGE (GMH/IVH): A COMBINED MRI AND HISTOLOGICAL APPROACH}

V. Supramaniam ${ }^{1}$, R. Vontell ${ }^{2}$, K. Amer ${ }^{1}$, L. Srinivasan ${ }^{1}$, J. Wyatt-Ashmead ${ }^{3}$, M. Rutherford ${ }^{1}$

${ }^{1}$ Perinatal Imaging Group, Imaging Sciences Department, ${ }^{2}$ Perinatal Imaging Group, Imaging Sciences Department/Institute of Reproductive and Developmental Biology, Clinical Sciences Centre, MRC, Imperial College London, ${ }^{3}$ Wigglesworth

Perinatal Pathology Services, St Mary's/

Hammersmith Hospital London, London, UK

Background: GMH/IVH remains a prevalent form of injury to immature brain. In the absence of overt complications, haemorrhage may result in mild ventriculomegaly and poor developmental outcome. The imaging appearances are suggestive of periventicular injury with subsequent tissue atrophy. Although essential for normal brain development, presence of resident MG may exacerbate the injury. We have shown increased activated MG in normally appearing PVWM compared with less susceptible 
regions. A rabbit model of $\mathrm{GMH} / \mathrm{IVH}$ demonstrated an increase in activated $M G$ in the adjacent PVWM, which may cause or exacerbate WM injury.

Aim: To use post mortem (PM) brain MRI and immunohistochemistry to investigate $M G$ distributions in the immature brain in the presence of $\mathrm{GMH} / \mathrm{IVH}$ and to compare to controls with normally appearing brain.

Methods: Preterm (< 30 wk GA) PM cases were recruited with parental consent and ethics approval. Images were acquired at 3T with T1 and T2 weighted sequences. Iba1 and CD45 MG were counted in the anterior and posterior PVWM areas, centrum semiovale, germinal matrix (GM), subplate and cortex.

Results: 6 brains showed isolated $\mathrm{GMH} / \mathrm{IVH}$ and 6 showed normal appearances. Total number of $M G$ in the $G M$ (lba1=14.21 $\pm 1.39 p<0.05$; CD45 $=11 \pm 4.70)$ and PVWM (Iba1 $=12.30 \pm 0.91$ $p<0.05$; CD45 $=4.8 \pm 1.52$ ) were higher than other regions in the $\mathrm{GMH} / \mathrm{IVH}$ group. GMH/IVH PVWM $M G$ numbers were higher than in the normal group $(p=0.005)$.

In the presence of IVH/GMH there was a marked increase in PVWM MG as seen in experimental models of IVH/GMH. These may be responsible for initiating or exacerbating an injurious process.

\section{8}

\section{DOPAMINE AND CIRCULATION IN THE BRAIN: A STUDY IN NEWBORN PIGLETS}

\author{
G.H. Hahn ${ }^{1}$, C. Heiring ${ }^{2}$, O. Pryds ${ }^{3}$, G. Greisen ${ }^{1}$ \\ ${ }^{1}$ Department of Neonatology, National University \\ Hospital, Rigshospitalet, Copenhagen, \\ ${ }^{2}$ Department of Paediatrics, Hillerød Hospital, \\ Hillerød, ${ }^{3}$ Department of Neonatology, National \\ University Hospital, Hvidovre Hospital, \\ Copenhagen, Denmark
}

BACKGROUND AND Aims: Dopamine is widely used to treat hypotension in newborns. Data regarding its effect on the circulation in the brain, however, are ambiguous. To test for a direct effect on the brain, we estimated the magnitude of variability in arterial blood pressure (ABP) passing from systemic to cerebral circulation (i.e. frequency gain) during an ABP-challenge with dopamine in piglets.
Methods: Piglets ( $n=7$, age 1-2 days) were anaesthetized and monitored with NIRS (cerebral oxygenation) during normo- and hypovolaemia. In both states, ABP was challenged with dopamine during a 20-minute stepwise dose-increment (20, $30,40$ and $50 \mu \mathrm{g} / \mathrm{kg} / \mathrm{min})$. Each infusion lasted $2 \frac{1}{2}$ minutes. Pauses lasting $2 \frac{1}{2}$ minutes were interpolated. Before and after the dopaminechallenge, ABP was challenged with six inflations of a thoracic aorta balloon. Each inflation lasted 30 seconds and pauses lasting 30 seconds were interpolated. Subsequently, frequency gain between ABP and cerebral oxygenation was calculated (frequency range: 0.003-0.04 Hz).

Results: Mean variability in ABP was $36 \pm 4.3 \mathrm{~mm} \mathrm{Hg}$ during balloon challenge and $17 \pm 3.2 \mathrm{~mm} \mathrm{Hg}$ during dopamine challenge (mean \pm SEM). Mean gain between ABP and cerebral NIRS was $0.11 \pm 0.019$ during $\mathrm{ABP}$-challenge with the aorta balloon and $0.12 \pm 0.029 \mu \mathrm{M} / \mathrm{mm} \mathrm{Hg}$ during ABP-challenge with dopamine $(P=0.86)$. The mean difference was 0.01 [95\%-Cl: -0.07 to +0.09 ]

Conclusions: ABP-variability induced by dopamine and by inflating an aortic balloon had similar effects on cerebral oxygenation. This suggests that dopamine has no direct constricting or dilating effect on the cerebral blood vessels or that such effects are counterbalanced by changes in cerebral oxygen uptake.

99

CORRELATION OF NON-INVASIVE
SYSTOLIC AND MEAN BLOOD
PRESSURE (BP) MEASUREMENTS WITH
ECHOCARDIOGRAPHIC HAEMODYNAMIC
ASSESSMENT

S. Gupta ${ }^{1}$, J.P. Wyllie ${ }^{2}$

${ }^{1}$ Neonatal Paediatrics, University Hospital of North Tees, Stockton on Tees, ${ }^{2}$ Neonatal Paediatrics, The James Cook University Hospital, Middlesbrough, UK

Objective: Systolic and mean $\mathrm{BP}$ values are routinely utilised to define hypotension on neonatal units. There is however no data correlating systolic or mean BP with cardiac haemodynamics in newborn infants.

Methods: In a randomised trial ${ }^{1}, 71$ infants received either two transfusions of $10 \mathrm{ml} / \mathrm{kg}$ or single transfusion of $20 \mathrm{ml} / \mathrm{kg}$. Clinical and echocardiographic haemodynamic measurements 